\title{
A NOVEL WEB APPLICATION (FOODCOACH) TO MONITOR AND IMPROVE EATING BEHAVIORS: PILOT RANDOMIZED CONTROLLED TRIAL
}

\author{
Diana Trojaniello, Francesca Sacchitelli, Elena Massa, Pietro Vismara and Alberto Sanna \\ Ospedale San Raffaele, Via Olgettina 60, Milan, Italy
}

\begin{abstract}
Nowadays, overweight and obesity are considered a global public and health problem. The use of ICT in weight management programs has been reported in improved long-term adherence and nutritional habits. Few studies validated ICT based approaches versus standard paper-based approaches. This study aimed at investigating the efficacy and acceptability of a novel Web App, i.e. FoodCoach, to guide and monitor dietary behaviours in an healthy sample population. A two arms randomized controlled trial study has been designed. Fourty healthy subjects have been included in two groups: the first group (control) obtained a standard paper-based not-personalized dietary intervention; the second group (experimental) obtained a novel Web-based personalized dietary intervention through the FoodCoach Web App. Both the groups were remotely followed for 40 days by expert dieticians, the first through telephone and emails, the second trough the Web App. At the start and the end of the 40-days experimental period, physical appointments with the dieticians and research team at the hospital premises have been organized. The results showed a significant weight loss, Body Mass Index and Fat Mass percentage reductions in the experimental group participants, while no differences in the control group subjects have been found. The acceptability of the proposed Web App was explored by means of questionnaire and engagement metrics (frequency of usage): a good level of engagement over the 40 days and high scores in terms of usability and user experience of the participants have been showed.
\end{abstract}

\section{KEYWORDS}

Nutritional Platform, Mediterranean Diet, Web Platform, E-Health, Remote Monitoring, User Experience

\section{INTRODUCTION}

Overweight and obesity are a global public health problem. About 2 billion of people worldwide are overweight and one third of them obese (Seidell and Halberstadt, 2015). In Italy, the Organisation for Economic Co-operation and Development (OECD) reports about 1 in 10 people is obese and OECD projections indicate that overweight rates will increase by a further $5 \%$ within ten years (OECD statistics, 2018). Wrong eating behaviors and a lack of physical activity are considered the primary causes.

Nowadays the gold standard treatment in clinical practice for overweight and obesity management consists of: a) a balanced diet prescribed by a nutrition expert (e.g. dietologist, dietitian); and b) a proper diet-daily assessment performed by both the dietitian and the patient itself, i.e. self-assessment (Naska, Lagiou and Lagiou, 2017). Several tools, such as 24-h dietary recall, Food Frequency Questionnaire FFQ (retrospective) and daily diet-diaries (prospective), have been recommended for dietary assessment. In particular, paper diet-diaries are commonly advocated as the gold standard tool for patient's eating behavior monitoring: guidelines recommend that patients provide a detailed account of timing, type and structure of everything eaten or drunk for at least three consecutive days (Burke et al., 2005). Studies showed that completing food diaries, in combination with dietary advice, is associated with weight loss (Kong et al., 2012). However, the paper diet-diaries are characterized by a low adherence (Glanz et al., 2006; Knudsen et al., 2011; Gondolf et al., 2012) mainly due to the burden associated to a) the food intake monitoring through a not automated tool and $b$ ) the memory effort requested. Therefore, the need of more engaging tools to improve diet diary filling usage and diet adherence is widespread.

Recent innovations in information and communication technology (ICT) offer new opportunities to overcome such limitations in diet management by providing new solutions for dietary assessment (Personal 
Digital Assistant, PDA, or Web-based diaries) and, at the same time, allowing experts to follow patients located in remote areas. Indeed the use of ICT tools in daily life activities is widespread among the population and, in this context, the diffusion of ICT tools, including mobile Health Applications (mHealth Apps), aimed to assist, inform and guide users in health issues management is promising (Ozdalga, Ozdalga and Ahuja, 2012). Therefore a growing interest has been registered in the use of ICT technologies to handle diet interventions: nutrition Apps for mobile devices (e.g., PDA, smartphones, tablets) are becoming increasingly accessible (Gilmore et al., 2014) and can assist the patients with the difficult task of intake recording for dietary assessment and self-monitoring (Lieffers and Hanning, 2012). The majority of commercially available nutritional mApps allow users to retrieve information on foods, track their food consumption and receive pills to lose weight or to improve nutritional behaviours. Indeed the use of ICT tools for personalized diet interventions has been found to improve adherence in diet-diary filling, increased awareness of the actual eating habits, help subjects in following dietary prescriptions and allow experts to remotely access to the patient's nutritional data (Probst and Tapsell, 2005). A recent study found that nutritional mApp use is also linked to significant short-term weight loss and that this weight loss is associated with adherence (Jacobs, Radnitz and Hildebrandt, 2017). On the other hand, nutritional mApps require users to actively engage with it in order to benefit and reach the personal objectives. Despite the proliferation of nutritional apps and their apparent potential in dietary interventions, there is currently limited evidence on the experience of using these ICT tool and how participants perceive the comparison between novel and traditional methods of dietary assessment (Carter et al., 2013; Jimoh et al., 2018). Indeed, the validation of the proposed ICT tools in terms of effectiveness, adherence and user engagement related to the dietary intervention against standard methodologies is desired. In addition, a stand-alone intervention (e.g. without the expert support), as that commonly proposed by the commercially available ICT tools, could be inappropriate (e.g. the patient could follow an unbalanced diet) and not effective.

We developed a responsive Web App for desktop and mobile devices which offers a Personalized Nutritional Plan (PNP) to guide users towards a healthy diet and behaviors, called FoodCoach (FC), by integrating Care-receiver (i.e. the patient) and Expert Care-giver (i.e. the nutrition expert) assessment and fostering Care-receiver reflective learning and self-management. The new developed tool allows the Care-receiver to: a) access the PNP established by the nutrition expert; b) track the food and beverages consumption; c) set periodical appointments (weigh yourself, appointment with the experts); d) self-monitor and track the progresses; e) access educational material provided by the experts; f) chat with the experts. At the same time, the proposed tool allows the Expert Care-giver to profile the Care-receiver, to check the automatically generated PNP, to validate the automatically generated dietary prescriptions and to remotely monitoring the Care-receiver progresses. The FC Web App has been benchmarked against commercially available systems, such as "A better plan (Microsoft HealthVault)", "Nutrino" and "SparkPeolple" , and contains a) a large, detailed food database (i.e. "DataFood") which includes the INRAN Italian database, the french CIQUAL database, and the american USDA one; b) weekly/daily personalized plan by Expert Care-giver; c) recipes for every suggested food including food pictures, nutritional details, ingredients and the descriptions; and d) the possibility to receive, in a quasi-real time manner, dietitian feedbacks and advices. These features have been added to engage users with the Web App in a real-life setting.

The current study aims to evaluate the efficacy, ease of use, acceptability, user engagement and perceived effectiveness of $\mathrm{FC}$ platform in providing a personalized nutritional intervention, for the diet management of a healthy population sample compared with a more traditional paper-based approach, through a two-arms randomized controlled pilot.

\section{MATERIALS AND METHODS}

\subsection{The FoodCoach Platform}

The FC platform can be described as a food recommendation system that uses a recipe dataset and annotations to recommend menus. It is a responsive web application for desktop and mobile devices and offers to patients a PNP prescribed by an expert dietitian, based on metabolic needs and preferences. It is based on a Front-Office portal addressed to patients and on a Back-Office one addressed to dietitians. Dietitians insert patients personal data (e.g. age, weight, BMI, nutritional habits, etc.), thus allowing the 
platform to properly compute the PNP and dietary suggestions. The FC platform (Back-Office) is characterized by the following sections:

- The Personalized Nutrition Plan (PNP) section through which the user accesses the PNP and consult the suggested meals everyday. The user may also choose between three different substitutions for any single meal. In the PNP are suggested some recipes;

- The Food Diary section which allows the user to daily record meals consumed, also being able to specify the quantity, the place and the time. It is also possible to indicate a missing meal and the motivation. In addition, it is also possible to track the daily water intake (Water filling);

- The Food Frequency Questionnaire (FFQ) section in which the user auto-fills a questionnaire to rebuild its eating habits, based on the frequency of consumption of a list of foods.

- The Statistics section allows the user to monitor the PNP adherence downloading reports that show progresses and body changes;

- The Educational Material section allows the user to check informative material related to eating behaviours suitable for a healthy and active lifestyle, such as information concerning nutrition;

- The Weight Ritual section allows the user to weekly update the body weight. The user also receives a weekly message as reminder for the body weight measurement. In this way the personal statistics remain updated and the clinical expert is able to monitor user's progresses;

- The Recipes section in which the user checks and chooses recipes whose ingredients correspond to the foods provided by the PNP for the suggested meals on the basis of the user's nutritional demand.

- The Chat section with the dietitian allows the user to get in touch with the dietitian directly, asking questions and doubts and getting clarifications.

In Figure 1 the landing page of the FC Web App has been showed.

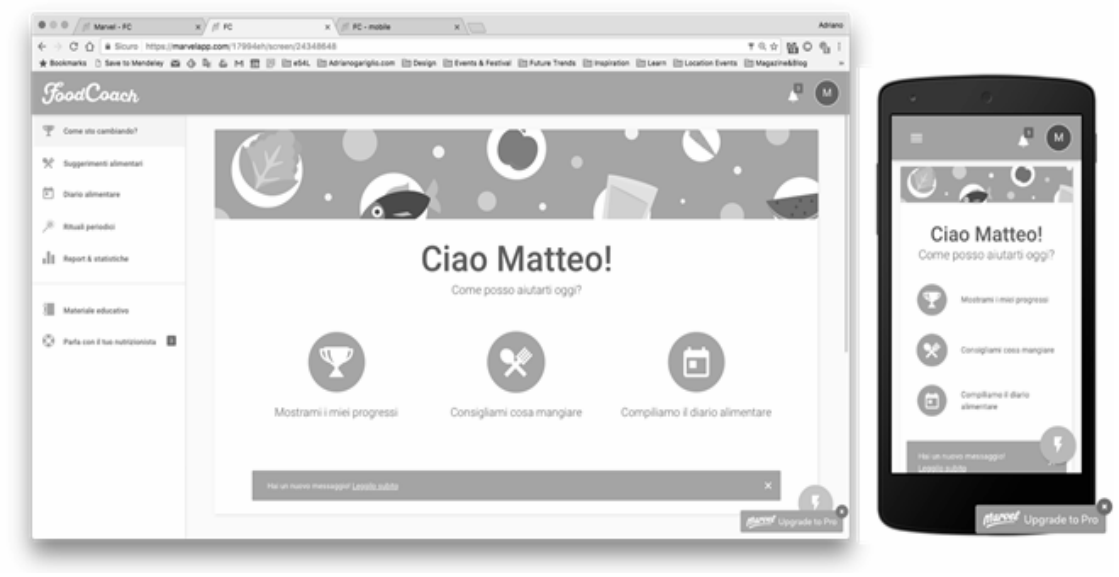

Figure 1. FC landing page: the responsiveness of the proposed Web App allows the users to access it through both PC and mobile devices (i.e. tablet, smartphone)

\subsection{Experimental Protocol}

The research has been conducted in Milan (Italy) at the San Raffaele Hospital (HSR) premises, from March 2017 to June 2017. The experimental protocol was approved by the HSR Ethical Committee (Protocol 653704-1 approved on 10/11/2018) and informed consent forms have been edited in accordance to the Declaration of Helsinki.

\subsubsection{Study Design and Randomisation}

The trial design was a single blind controlled parallel 2-arm randomized pilot intervention trial (see Figure 2) undertaken between March and June 2017. Eligible participants were randomised in a 1:1 ratio to either "experimental" $(\mathrm{n}=20)$ or "control" group $(\mathrm{n}=20)$. The randomisation sequence was generated by a person unrelated to the project. As a pilot trial, the primary outcomes were the users' engagement, acceptance and adherence to the intervention (frequency of use). Secondary outcomes were anthropometric measures. Once included in the study, participants $(\mathrm{n}=40)$ have been invited at HSR facilities to attend a first baseline 
assessment. During the baseline assessment, the participants were assigned to one of the groups (experimental or control) according to the randomization list. Weight, height, Body Mass Index, Fat Mass percentage and body circumferences have been measured during a standard nutritional visit. Baseline self-completed questionnaire was also provided to collect information on demographics and food consumption habits, i.e. Food Frequency Questionnaire (FFQ). For the experimental group participants, the dietician created a FC user profile along with a PNP. Credentials were sent by email to the participant to access the FC platform.

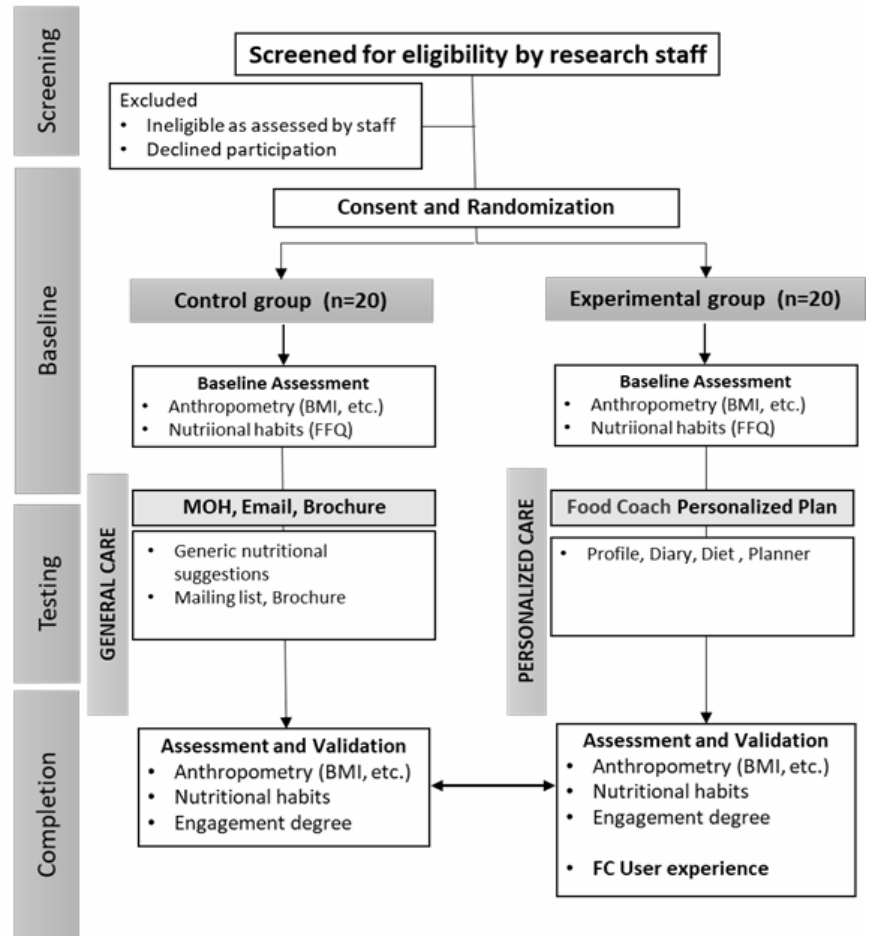

Figure 2. Flow of participants through a randomized 2 arms pilot trial of the FC Web App (N=40)

Participants were then instructed for the experimental period activities according to the belonging group:

(a) Control Group (CG): 40-days "not-personalized" intervention consisting in (a) to follow the nutritional guidelines (INRAN guidelines) provided by the nutritionist twice a week via email; b) to fill the paper diet-diary daily; c) to register their weight weekly and insert the information in the paper diet-diary.

(b) Experimental Group (EG): 40-days "personalized" intervention consisting in (a) to follow the PNP daily provided by FC; b) to fill the FC food diary daily; c) to register their weight weekly and insert the information in the weight ritual section.

Participants enrolled in the EG were then introduced by the research team to the FC platform usage to assess the usability of the platform. At the end of the baseline assessment, EG participants were asked to fill the System Usability Scale (SUS) checklist to rank the FC usability.

After the 40-days experimental period, both CG and EG participants underwent to a second appointment at HSR facilities (final assessment) where received a second nutritional visit. Weight, height, Body Mass Index, Fat Mass percentage and body circumferences have been measured again. Final self-completed questionnaire was also provided, i.e. FFQ. At the end of the final assessment, EG participants were asked to fill questionnaire about user engagement and user experience to assess the FC user experience (UX).

\subsubsection{Recruitment Strategy}

Participants were recruited among HSR workers and relatives by advertising through email, intranet and brochures. Advertising material encouraged participants to contact the research team for the preliminary screening. The eligibility criteria were: absence of metabolic diseases, food allergies or any pathology 
directly related to nutrition; a value of Body Mass Index (BMI) higher than 29.9; aged 18 to 65 years; willing to commit the necessary time and effort to the study; not pregnant, breast-feeding or planning a pregnancy; no involvement in other weight management program; able to read and write in Italian; able to access Internet; willing to be randomized to 1 of the two groups.

A total of 355 subjects were interested in participating to this research. From them, 89 were screened to fulfill the inclusion criteria and 40 subjects in total were enrolled. 49 have been excluded due to the presence of food allergies, metabolic diseases, and for intestinal, liver and kidney diseases.

\subsubsection{Interventions}

The activities took place both at HSR premises and at participants home. For each participant, two visits at the hospital, one at the beginning and one at the end of the study period (40 days), were scheduled. The experimental period lasted 40 days in order to ensure an adequate range of time allowing the subjects to adhere to the nutritional plan suggested both in personalized (EG) and not personalized (CG) intervention. The subjects were visited at the start and at the end of the experimental period by a registered dietitian. During the 40 days, the subjects were asked to carefully follow the given nutritional suggestions, both personalized and not personalized. At the end of the experimental period, they were invited at HSR to undergo a second nutritional visit and to be evaluated in terms of user engagement degree and UX.

\subsubsection{Assessment Tools}

a) Nutritional assessment (both $\mathrm{CG}, \mathrm{EG}$ )

- Anthropometric Indices: BMI, Fat Mass percentage (\%FM) and Weight (W) were considered to evaluate body changes in between the start and the end of the experimentation.

b) Usability assessment (only EG)

- System Usability Scale (SUS) (Brooke, 1996) a simple, ten-item Likert scale giving a global view of subjective assessments of usability. It is generally used after the respondent has had an opportunity to use the system being evaluated.

c) User engagement assessment (only EG)

- Frequency of use (FoU): the rate of usage (weekly and overall) of the FC functionalities: Food Diary (and Water filling), Weight Ritual, Chat.

- User Engagement Questionnaire (O’Brien and Toms, 2010): a multidimensional scale to measure user engagement based on six attributes of user engagement (Perceived Usability, Aesthetics, Focused Attention, Felt Involvement, Novelty, and Endurability)

d) User experience assessment (only EG)

- $\quad$ Standardized User Experience Percentile Rank Questionnaire (SUPR-Q) (Sauro, 2015): 8 item questionnaire for measuring the quality of the website UX. It is a 5-point rating scale to measure perceptions of Usability, Trust, Credibility, Appearance and Loyalty for Websites.

\subsection{Data Analysis}

- Personalized intervention efficacy (both $\mathrm{CG}, \mathrm{EG}$ )

First of all, a Shapiro test for data normality check has been done on the anthropometric indices. Mean (m) and standard deviation (sd) of the anthropometric indices collected during the first $\left(\mathrm{W}_{\mathrm{IN}}, \mathrm{BMI}_{\mathrm{IN}}, \% \mathrm{FM} \mathrm{MN}_{\mathrm{IN}}\right)$ and second ( $\mathrm{W}_{\text {out }}, \mathrm{BMI}_{\mathrm{OUT}}, \% \mathrm{FM}_{\mathrm{OUT}}$ ) visits have been computed for each group. A T-test between IN and OUT measures for each group (CG, EG) has been done to verify if significant differences were present after the 40-days studies in one or both the two groups.

- Usability, User Engagement and Experience (only EG)

Usability - According to the level of agreement selected for each statement of the SUS questionnaire, a SUS score has been computed for each participant. According to (Bangor, Kortum and Miller, 2009) depending on whether the reported SUS score was greater or smaller than 68, the FC platform was defined usable or not.

User Engagement - The FoU has been computed for each FC section (Food Diary and Water filling, Weight Ritual, Chat) for each participant as the percentage of no use, less of $50 \%$ and more than $50 \%$ usage. In addition, the weekly usage for the experimental period ( 6 weeks) has been computed. The user engagement has been also explored by means of a specific questionnaire, i.e. the user engagement questionnaire: for each questionnaire dimension (Perceived Usability, Aesthetics, Focused Attention, Felt Involvement, Novelty, and Endurability) mean values and standard deviations have been computed among the EG participants. 
User experience $(U X)$ : the user experience has been explored by means of the $S U P R-Q$ : for each questionnaire dimension (Usability, Trust, Credibility, Appearance and Loyalty) mean values and standard deviations have been computed among the EG participants.

\section{RESULTS}

In the following, the main results of the study have been provided.

\subsection{Population Characteristics}

Subjects' characteristics (Age, Gender, Occupation) are reported in Table I. Among the fourty subjects recruited for the study (20 in EG, 20 in CG), 4 subjects from the CG and 2 subjects from the EG dropped out. Characteristics and data of drop-out subjects have not been included in the following table.

Table 1. Characteristics of the participants enrolled in the 2 -arms pilot

\begin{tabular}{lcc}
\hline & CG $(\mathbf{n}=\mathbf{1 6})$ & EG $(\mathbf{n = 1 8})$ \\
\hline Age [m (sd)] & $43,7(9,4)$ & $39(11)$ \\
Gender (female) [\%] & $37,5 \%$ & $61,1 \%$ \\
Occupation, employee [\%] & $18,7 \%$ & $33,3 \%$ \\
\hline
\end{tabular}

\subsection{Personalized Intervention Efficacy}

Anthropometric indices at the baseline $\left(\mathrm{W}_{\mathrm{IN}}, \mathrm{BMI}_{\mathrm{IN}}, \% \mathrm{FM}_{\mathrm{IN}}\right)$ and final ( $\mathrm{W}_{\mathrm{OUT}}, \mathrm{BMI}_{\mathrm{OUT}}$, \% FM $\mathrm{OUT}$ ) assessments have been reported in Table 2 along with the results of the T-test performed for each antropometric index by comparing the baseline and the final measure for both the CG and EG.

Table 2. Baseline (IN) and final (OUT) anthropometric indices and the p-value obtained for each IN-OUT comparison

\begin{tabular}{|c|c|c|c|c|c|c|}
\hline & \multicolumn{3}{|c|}{ CG } & \multicolumn{3}{|c|}{ EG } \\
\hline & $m$ & $s d$ & $p$-value & $m$ & $s d$ & $p$-value \\
\hline $\mathrm{W}_{\mathrm{IN}}$ & 74,5 & 12,7 & \multirow{2}{*}{ ns } & 72,6 & 11,3 & \multirow{2}{*}{$<0.01$} \\
\hline Wout & 74,0 & 12,6 & & 71,1 & 11,2 & \\
\hline $\mathrm{BMI}_{\mathrm{IN}}$ & 25,4 & 3,0 & \multirow{2}{*}{ ns } & 24,8 & 2,7 & \multirow{2}{*}{$<0.01$} \\
\hline BMIout & 25,2 & 3,1 & & 24,2 & 2,5 & \\
\hline$\% \mathrm{FM}_{\mathrm{IN}}$ & 25,7 & 7,0 & \multirow{2}{*}{ ns } & 25,1 & 7,5 & \multirow{2}{*}{$<0.05$} \\
\hline$\%$ FMout & 25,2 & 7,1 & & 24,0 & 7,3 & \\
\hline
\end{tabular}

Significative differences $(\mathrm{p}<0.05)$ were found for all the anthropometric indices IN-OUT in the EG, while no differences have been found in the CG data.

\subsection{Usability, User Engagement and User Experience}

\subsubsection{Usability}

The SUS scores obtained in the EG group are the following: the $89 \%$ of participants rated it as usable (SUS $>68$ ) while the remaining $11 \%$ rated it unsuable (SUS $\leq 68$ ).

\subsubsection{User Engagement}

In Figure 3, Figure 4, Figure 5, Figure 6 both the overall and weekly FoU for each FC section have been reported. The results of the User Engagement questionnaire have been reported in Figure 7.

\subsubsection{User Experience}

The Figure 8 shows the SUPR-Q questionnaire results averaged on EG participants. 


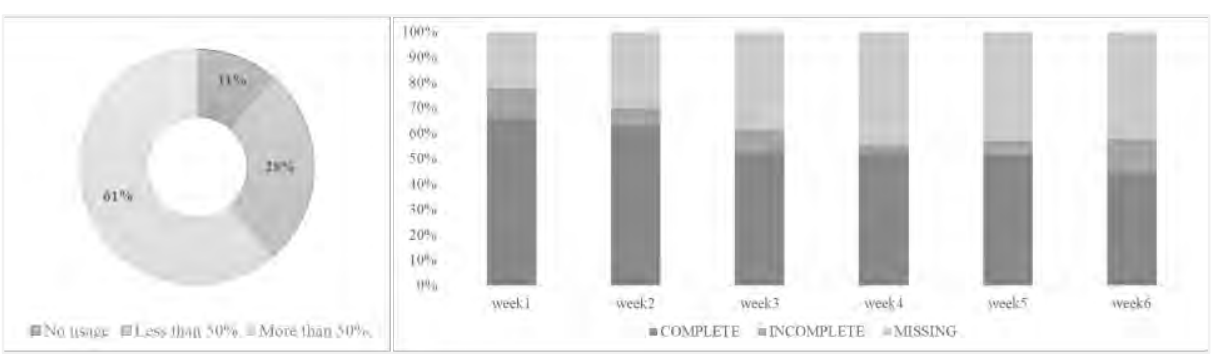

Figure 3. (a) Diary overall frequency of usage; (b) Diary weekly frequency of usage

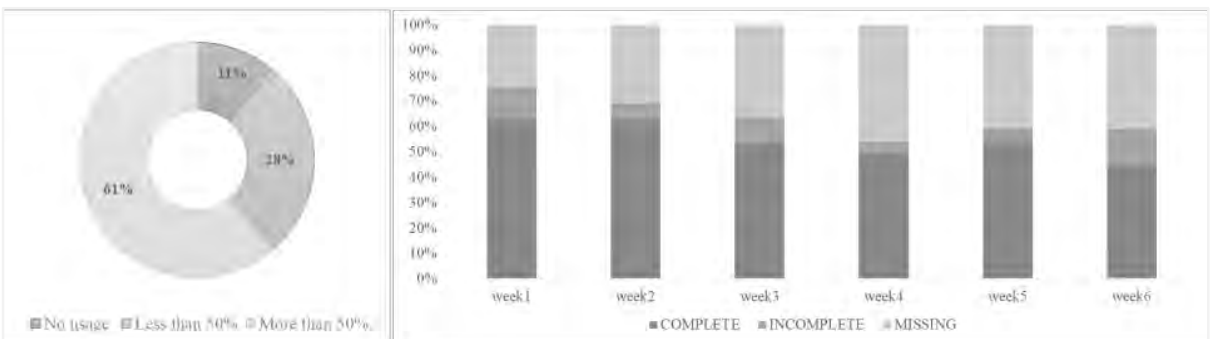

Figure 4. (a) Water filling overall frequency of usage; (b) Water filling weekly frequency of usage
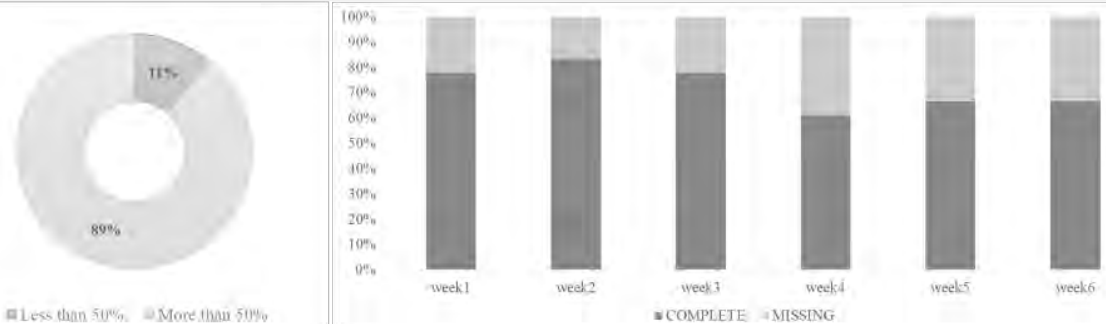

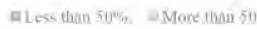

Figure 5. (a) Weight ritual overall frequency of usage; (b) Weight ritual weekly frequency of usage

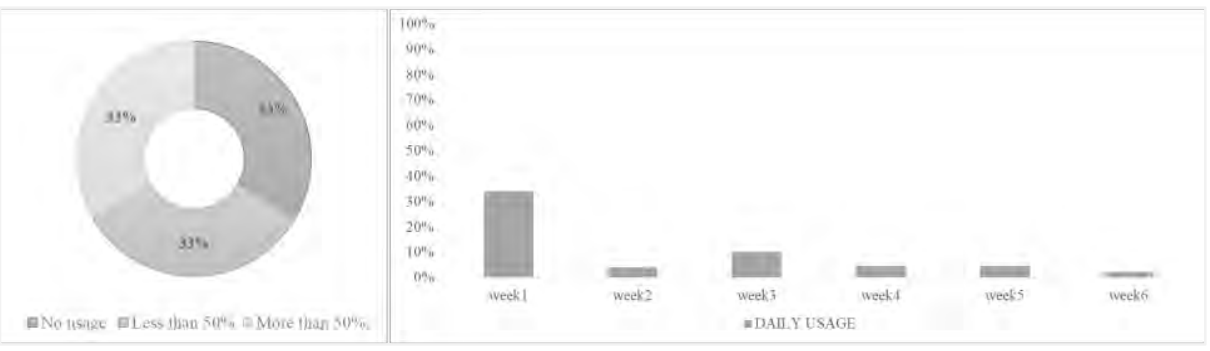

Figure 6. (a) Chat overall frequency of usage; (b) Chat daily frequency of usage per week

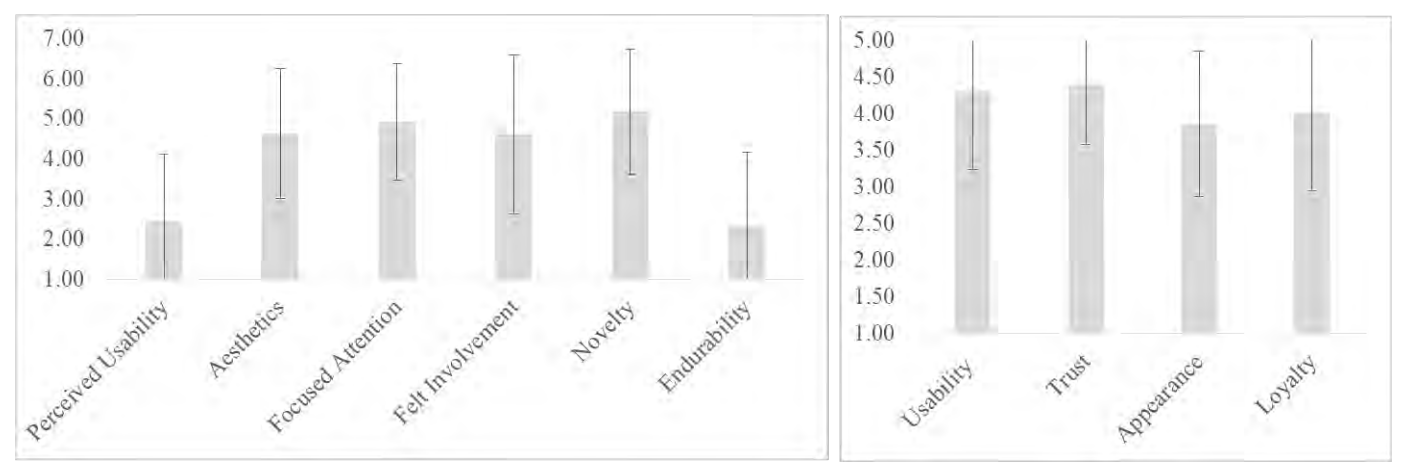

Figure 7. User engagement scores averaged on EG participants

Figure 8. SUPR-Q scores averaged on EG participants 


\section{DISCUSSION AND CONCLUSION}

The current study showed that the FC Web App could be a feasible and acceptable instrument for monitoring and improving eating habits in the sample population. In terms of recruitment, about 9 times $(n=355)$ the target number of subjects $(n=40)$ showed interest in partecipating in the study.

E-mail communication was the most successful recruitment strategy. Overall, the trial suffered of $15 \%$ attrition rate: among the 6 subjects who abandoned the trial, 2 belonged to the EG and 4 to the CG, i.e. $50 \%$ more subjects dropped out in CG. Drop out may reflect a person's dissatisfaction with the intervention and unequal dropout among groups is likely to be intervention-related. Attrition rate in the EG (10\%) seems to be lower with respect to other studies specifically based on Web-based interventions (Neve et al., 2010; Carter et al., 2013) where an attrition rate of $20 \%$ has been found. Although the pilot trial was not statistically powered to detect a difference in weight change among the groups, it provided some data on effect sizes within each group. In fact, CG showed a mean weight reduction of $0.5 \pm 0.1 \mathrm{Kg}$, while the EG showed a mean weight reduction of $1.5 \pm 0.1 \mathrm{Kg}$. The same happened for the BMI and Fat Mass Percentage: a reduction of $0.2 \pm 0.1$ (BMI) and $0.5 \pm 0.1 \%(\mathrm{FM})$ characterized the $\mathrm{CG}$ while the EG was characterized by a reduction of $0.6 \pm 0.2$ (BMI) and $1.1 \pm 0.2 \%(\mathrm{FM})$. The t-test carried out to compare the IN-OUT data for each group showed a statistical difference only in the EG, for all the antropometric indices (W, BMI, FM\%). For the EG, the SUS score showed an high perceived usability of the platform, i.e. only 2 over 18 subjects reported a SUS score lower than 68. After the 40-days trial, the user engagement of the EG participants has been evaluated both by analysing the actual frequency of usage of the various FC sections and by collecting subjective feedbacks on the overall usage over time from the participants. Both the results showed that a high, both objective and subjective, engagement rate. The "Diary" and "Water filling" sections have been used, at least one time, by 16 over 18 participants $(89 \%)$. The "Weight ritual" section has been used at least once by all the participants and by 16 over $18(89 \%)$ for more than 3 weeks. The "Chat" section has been used at least one by 12 participants $(66 \%)$ while only $33 \%$ used the function for more than $50 \%$ of the trial period. The frequency of usage of the various sections showed that all the FC functionalities have been used at least one time by the majority of the subjects. The rate over the weeks has been also analysized to understand if any engagement loss trend was present: only the "Chat" section showed a significant higher usage during the first week (34\%) with respect to the last one (2\%). This result is quite expected since the main doubts or questions are likely to arise in the first period more than the last one. Regarding the Diary, Water filling and Weight ritual no specific usage trends have been observed during the trial period with exception to an overall (complete + incomplete pages) FoU reduction from the first to the last week of $10 \%$ for the Diary (from $68 \%$ to $58 \%$ ) and of $6 \%$ for the Water filling (from $75 \%$ to $59 \%$ ) sections. Engagement data obtained through the questionnaire analysis showed high levels of novelty, focused attention and felt involvment. Instead, the perceived usability after 40-days of usage showed an overall low score: this means that the daily usage of FC has been perceived less usable than expected (i.e. comparing with the pre-usage SUS). Finally, the high scores obtained in the UX questionnaire showed an overall acceptable UX after the 40-days of usage.

The present study showed some limitations: the sample group was limited; the FC Web App was a prototype and participants reported that they encountered bugs, thus sometimes affecting their engagement; participants knew what interventions were available and when included in the CG the engagement was lower.

In conclusion, this preliminary study showed a good level acceptance and efficacy of the FC Web platform with respect to a not-personalized standard diet intervention. As future studies, the adherence to the suggested personalized (PNP) and not personalized (INRAN guidelines) intervention by analysing the food diaries (both paper based and Web based) data will be explored. In addition, a comparison analysis of the eating behaviors as collected through the FFQ understand the compliance to the Mediterranean Diet, before and after the intervention.

\section{ACKNOWLEDGEMENT}

The research leading to these results has received funding from the European Union's Horizon 2020 research and innovation programme under grant agreement $n^{\circ} 653704-$ OPERANDO. 


\section{REFERENCES}

Bangor, A., Kortum, P. and Miller, J. (2009) 'Determining what individual SUS scores mean: Adding an adjective rating scale', Journal of usability studies, 4(3), pp. 114-123. doi: 66.39.39.113.

Brooke, J. (1996) 'SUS-A Quick and Dirty Usability Scale'. doi: 10.1002/hbm.20701.

Burke, L. E., Warziski, M., Starrett, T., Choo, J., Music, E., Sereika, S., Stark, S. and Sevick, M. A. (2005) 'Self-monitoring dietary intake: current and future practices.', Journal of renal nutrition: the official journal of the Council on Renal Nutrition of the National Kidney Foundation. United States, 15(3), pp. 281-290.

Carter, M. C., Burley, V. J., Nykjaer, C. and Cade, J. E. (2013) 'Adherence to a smartphone application for weight loss compared to website and paper diary: pilot randomized controlled trial', Journal of medical Internet research. JMIR Publications Inc., 15(4), pp. e32-e32. doi: 10.2196/jmir.2283.

Gilmore, L. A., Duhé, A. F., Frost, E. A. and Redman, L. M. (2014) 'The technology boom: a new era in obesity management', Journal of diabetes science and technology. SAGE Publications, 8(3), pp. 596-608. doi: 10.1177/1932296814525189.

Glanz, K., Murphy, S., Moylan, J., Evensen, D. and Curb, J. D. (2006) 'Improving dietary self-monitoring and adherence with hand-held computers: a pilot study.', American journal of health promotion: AJHP. United States, 20(3), pp. 165-170. doi: 10.4278/0890-1171-20.3.165.

Gondolf, U. H., Tetens, I., Hills, A. P., Michaelsen, K. F. and Trolle, E. (2012) 'Validation of a pre-coded food record for infants and young children.', European journal of clinical nutrition. England, 66(1), pp. 91-96. doi: 10.1038/ejen.2011.133.

Jacobs, S., Radnitz, C. and Hildebrandt, T. (2017) 'Adherence as a predictor of weight loss in a commonly used smartphone application.', Obesity research \& clinical practice. Netherlands, 11(2), pp. $206-214$. doi: 10.1016/j.orcp.2016.05.001.

Jimoh, F., Lund, E. K., Harvey, L. J., Frost, C., Lay, W. J., Roe, M. A., Berry, R. and Finglas, P. M. (2018) 'Comparing Diet and Exercise Monitoring Using Smartphone App and Paper Diary: A Two-Phase Intervention Study', JMIR mHealth and uHealth. JMIR Publications, 6(1), pp. e17-e17. doi: 10.2196/mhealth.7702.

Knudsen, V. K., Gille, M.-B., Nielsen, T. H., Christensen, T., Fagt, S. and Biltoft-Jensen, A. (2011) 'Relative validity of the pre-coded food diary used in the Danish National Survey of Diet and Physical Activity.', Public health nutrition. England, 14(12), pp. 2110-2116. doi: 10.1017/S1368980011001650.

Kong, A., Beresford, S. A. A., Alfano, C. M., Foster-Schubert, K. E., Neuhouser, M. L., Johnson, D. B., Duggan, C., Wang, C.-Y., Xiao, L., Jeffery, R. W., Bain, C. E. and McTiernan, A. (2012) 'Self-monitoring and eating-related behaviors are associated with 12-month weight loss in postmenopausal overweight-to-obese women.', Journal of the Academy of Nutrition and Dietetics. United States, 112(9), pp. 1428-1435. doi: 10.1016/j.jand.2012.05.014.

Lieffers, J. R. L. and Hanning, R. M. (2012) 'Dietary assessment and self-monitoring with nutrition applications for mobile devices.', Canadian journal of dietetic practice and research : a publication of Dietitians of Canada $=$ Revue canadienne de la pratique et de la recherche en dietetique: une publication des Dietetistes du Canada. Canada, 73(3), pp. e253-60. doi: 10.3148/73.3.2012.e253.

Naska, A., Lagiou, A. and Lagiou, P. (2017) 'Dietary assessment methods in epidemiological research: current state of the art and future prospects', F1000Research. F1000Research, 6, p. 926. doi: 10.12688/f1000research.10703.1.

Neve, M., Morgan, P. J., Jones, P. R. and Collins, C. E. (2010) 'Effectiveness of web-based interventions in achieving weight loss and weight loss maintenance in overweight and obese adults: a systematic review with meta-analysis.', Obesity reviews : an official journal of the International Association for the Study of Obesity. England, 11(4), pp. 306-321. doi: 10.1111/j.1467-789X.2009.00646.x.

O'Brien, H. L. and Toms, E. G. (2010) 'The development and evaluation of a survey to measure user engagement in e-commerce environments', Journal of the American Society for Information Science \& Technology, 61(1), pp. 50-69. doi: 10.1002/asi.21229.1.

OECD statistics (2018). Available http://www.oecd.org/els/ health-systems/obesityandtheeconomicsofpreventionfitnotfat-italykeyfacts.htm (Accessed: 17 January 2019).

Ozdalga, E., Ozdalga, A. and Ahuja, N. (2012) 'The smartphone in medicine: a review of current and potential use among physicians and students.', Journal of medical Internet research. Canada, 14(5), p. e128. doi: 10.2196/jmir.1994.

Probst, Y. C. and Tapsell, L. C. (2005) 'Overview of computerized dietary assessment programs for research and practice in nutrition education.', Journal of nutrition education and behavior. United States, 37(1), pp. 20-26.

Sauro, J. (2015) 'SUPR-Q: A Comprehensive Measure of the Quality of the Website User Experience', Journal of Usability Studies, 10(2), pp. 68-86. Available at: http://www.upassoc.org.

Seidell, J. C. and Halberstadt, J. (2015) 'The global burden of obesity and the challenges of prevention.', Annals of nutrition \& metabolism. Switzerlanworld, 66 Suppl 2, pp. 7-12. doi: 10.1159/000375143. 